PROCEEDINGS OF THE

AMERICAN MATHEMATICAL SOCIETY

Volume 135, Number 10, October 2007, Pages 3255-3262

S $0002-9939(07) 08902-2$

Article electronically published on May 2, 2007

\title{
RATE OF $L^{2}$-CONCENTRATION OF BLOW-UP SOLUTIONS FOR CRITICAL NONLINEAR SCHRÖDINGER EQUATION
}

\author{
XIAOGUANG LI AND JIAN ZHANG \\ (Communicated by David S. Tartakoff)
}

\begin{abstract}
This paper concerns the rate of $L^{2}$-concentration of the blowup solutions for the critical nonlinear Schrödinger equation. The result of Tsutsumi is improved in terms of Merle and Raphaël's recent arguments.
\end{abstract}

\section{INTRODUCTION}

This paper is concerned with the Cauchy problem of the following nonlinear Schrödinger equation:

$$
\begin{array}{r}
i u_{t}+\triangle u+|u|^{4 / N} u=0, \quad t \geq 0, x \in \mathbb{R}^{N}, \\
u(0, x)=u_{0}, \quad x \in \mathbb{R}^{N},
\end{array}
$$

where $u=u(t, x):[0, T) \times R^{N} \rightarrow C$ and $0<T \leq \infty ; i=\sqrt{-1} ; \triangle$ is the Laplace operator on $\mathbb{R}^{N}$. If we replace the nonlinear term by $|u|^{p-1} u$, it is known that the exponent $p=p_{c}=1+4 / N$ in dimension $N$ is the critical value for nonexistence of global solutions (see [2, 20]).

For the Cauchy problem (1.1), (1.2), Ginibre and Velo [3] established the local existence in $H^{1}\left(\mathbb{R}^{N}\right)$. Glassey [4, Weinstein [20], Ogawa and Tsutsumi [17, Zhang 21. proved that for some initial data, the solutions of the Cauchy problem (1.1), (1.2) blow up in finite time.

In particular, let $Q(x)$ be the ground state (see [5, 18]), which is the unique, positive, radially symmetric solution of the following nonlinear elliptic equation:

$$
-\triangle u+u-|u|^{4 / N} u=0, \quad u \in H^{1}\left(\mathbb{R}^{N}\right) .
$$

Weinstein [20] proved that if $\left\|u_{0}\right\|_{L^{2}}<\|Q\|_{L^{2}}$, then the solutions of the Cauchy problem (1.1), (1.2) globally exist. On the other hand, if $\left\|u_{0}\right\|_{L^{2}} \geq\|Q\|_{L^{2}}$, then the solutions of the Cauchy problem (1.1), (1.2) may blow up.

The ground state $Q$ of equation (1.3) plays an important role in the formation of singularities for the solutions of the Cauchy problem (1.1), (1.2). Merle and

Received by the editors July 26, 2006.

2000 Mathematics Subject Classification. Primary 35Q55; Secondary 35Q51, 35B05.

Key words and phrases. Critical nonlinear Schrödinger equation, blow up, rate of $L^{2}$ concentration.

The first author is partially supported by the National Science Foundation of the People's Republic of China (No. 10271084).

The second author is partially supported by the National Science Foundation of the People's Republic of China (No. 10271084).

(C)2007 American Mathematical Society Reverts to public domain 28 years from publication 
Tsutsumi [12] studied the behavior of the radially symmetric blow-up solutions of the Cauchy problem (1.1), (1.2) and obtained the following result:

Suppose that $u_{0}=u_{0}(|x|), N \geq 2$ and that the solution $u(t) \in C\left([0, T), H^{1}\right)$ of the Cauchy problem (1.1), (1.2) blows up at $T$. Then for all $R>0$, one has

$$
\liminf _{t \rightarrow T}\|u(t)\|_{L^{2}(|x|<R)} \geq\|Q\|_{2},
$$

where $Q$ is the ground state solution of (1.3).

Under the same conditions, Tsutsumi [19] further showed that for any $\varepsilon>0$, there exists a $K>0$ such that

$$
\liminf _{t \rightarrow T}\|u(t)\|_{\left.L^{2}\left(|x|<K(T-t)^{1 / 2}\right)\right)} \geq(1-\varepsilon)\|Q\|_{2} .
$$

(1.4) shows that under the condition that the initial datum is radially symmetric, the $L^{2}$-density of the blow-up solution of the Cauchy problem (1.1), (1.2) $u(t, x)$ concentrates at the origin 0 as $t \rightarrow T$ (blow-up time). We call this phenomenon $L^{2}$-concentration or mass concentration. Furthermore, (1.5) gives the rate of $L^{2}$ concentration.

In recent years, Merle and Raphaël studied the blow-up solutions with small super critical mass, i.e.,

$$
u_{0} \in B_{\alpha^{*}}=\left\{u_{0} \in H^{1} \quad \text { with } \int Q^{2} \leq \int u_{0}^{2}<\int Q^{2}+\alpha^{*}\right\},
$$

for some parameter $\alpha^{*}$ small enough. They obtained a series of profound results on the qualitative properties such as the blow-up rate and $L^{2}$-concentration ([7]-[11]). In particular, Merle and Raphaël established the sharp upper and lower bound of the blow-up rate in 17, 11. In terms of the sharp lower bound of the blow-up rate we studied the rate of $L^{2}$-concentration for the blow-up solutions, and (1.5) is improved; that is, under some conditions, we can show that for any $\varepsilon>0$, there exists a $K>0$ such that:

$$
\liminf _{t \rightarrow T}\|u(t)\|_{L^{2}\left(|x|<K\left(\frac{T-t}{\ln |\ln (T-t)|}\right)^{\frac{1}{2}}\right)}>(1-\varepsilon)\|Q\|_{L^{2}} .
$$

This paper is organized as follows. In Section 2, we recall the lower bound of the blow-up rate recently obtained by Merle and Raphaël. In Section 3, we investigate the rate of $L^{2}$-concentration.

We conclude this section with some notation. We denote $L^{q}\left(\mathbb{R}^{N}\right)$ and $\|\cdot\|_{L^{q\left(\mathbb{R}^{N}\right)}}$ by $L^{q}$ and $\|\cdot\|_{q}$, respectively. The various positive constants will be simply denoted by $C$.

\section{BLOW-UP RATE}

From [2] or [3] one has the following proposition.

Proposition 2.1. For any $u_{0} \in H^{1}$, there exists a unique solution $u(t, x)$ of the Cauchy problem (1.1), (1.2) in $C\left([0, T) ; H^{1}\right)$ for some $T \in(0, \infty]$ (maximal existence time), and $u(t)$ satisfies the two conservation laws of mass and energy:

$$
\begin{gathered}
\|u(t)\|_{2}=\left\|u_{0}\right\|_{2}, \\
E(u(t)):=\|\nabla u(t)\|_{2}^{2}-\frac{1}{1+2 / N}\|u(t)\|_{2+4 / N}^{2+4 / N}=E\left(u_{0}\right)
\end{gathered}
$$

for $t \in[0, T)$. Furthermore, we have the following alternatives: $T=\infty$ (global existence) or else $T<\infty$ and $\lim _{t \rightarrow T}\|u(t)\|_{H^{1}}=\infty$ (blow up). 
The blow-up rate of the Cauchy problem (1.1), (1.2) is very complicated:

- There exist in dimension $N=2$ solutions with blow-up rate $\|\nabla u(t)\|_{2} \sim \frac{1}{T-t}$ (see [1]).

- Another fact suggested by numerical simulations is the existence of solutions blowing up as $\|\nabla u(t)\|_{2} \sim\left(\frac{\ln |\ln (T-t)|}{T-t}\right)^{\frac{1}{2}}$ (see [6]).

For the blow-up solutions with small super critical data $\left(u_{0} \in B_{\alpha^{*}}\right)$, Merle and Raphaël established the shape lower bound for the blow-up rate in [11. The proof of Merle and Raphaël [11 depends on the following property.

Spectral Property. Let $N \geq 1$. Consider the Schrödinger operators

$$
E_{1}=-\Delta+\frac{2}{N}\left(\frac{4}{N}+1\right) Q^{\frac{4}{N}-1} y \cdot \nabla Q, \quad E_{2}=-\Delta+\frac{2}{N} Q^{\frac{4}{N}-1} y \cdot \nabla Q,
$$

and the real-valued quadratic form for $\varepsilon=\varepsilon_{1}+i \varepsilon_{2} \in H^{1}$ :

$$
H(\varepsilon, \varepsilon)=\left(E_{1} \varepsilon_{1}, \varepsilon_{1}\right)+\left(E_{2} \varepsilon_{2}, \varepsilon_{1}\right) .
$$

Then there exists a universal constant $\delta>0$ such that $\forall \varepsilon \in H^{1}$, if $\left(\varepsilon_{1}, Q\right)=$ $\left(\varepsilon_{1}, Q_{1}\right)=\left(\varepsilon_{1}, y Q\right)=\left(\varepsilon_{2}, Q_{2}\right)=\left(\varepsilon_{2}, \nabla Q\right)=0$, then

$$
H(\varepsilon, \varepsilon) \geq \delta\left(\int|\nabla \varepsilon|^{2} d x+\int|\varepsilon|^{2} e^{-|y|} d x\right),
$$

where $Q_{1}=\frac{N}{2} Q+y \cdot \nabla Q$ and $Q_{2}=\frac{N}{2} Q_{1}+y \cdot \nabla Q_{1}$.

Remark 2.2. This property has been proved in 9] for dimension $N=1$ using the explicit formula for the ground state $Q$. In dimensions $N=2,3,4$, this property accords with the numerical computation.

Proposition 2.3 (Merle and Raphaël [1]). Let $N=1$ or $N \geq 2$ assuming that the Spectral Property holds true. There exists $\alpha^{*}>0$ and a universal constant $C^{*}>0$ such that the following is true. Let

$$
\int Q^{2} \leq \int u_{0}^{2}<\int Q^{2}+\alpha^{*}
$$

and assume that the corresponding solution $u(t)$ of the Cauchy problem (1.1), (1.2) blows up in finite time, $0<T<+\infty$. Then one has the following lower bound on the blow-up rate for $t$ close to $T$ :

$$
\|\nabla u(t)\|_{2} \geq C^{*}\left(\frac{\ln |\ln (\tau-t)|}{\tau-t}\right)^{\frac{1}{2}} .
$$

\section{RATE OF $L^{2}$-CONCENTRATION}

Now, we state the main theorem of this paper:

Theorem 3.1. Suppose all the assumptions in Proposition 2.3 are satisfied. Let $N \geq 2$, and let $u(t)$ be a radially symmetric solution of the Cauchy problem (1.1), (1.2) in $C([0, T) ; H)$ such that $u(t)$ blows up at a finite time $T$.

(i) If $a(t)$ is a decreasing function from $[0, T)$ to $R^{+}$such that $a(t) \longrightarrow 0(t \rightarrow T)$ and $\frac{\left(\frac{\ln |\ln (T-t)|}{T-t}\right)^{\frac{1}{2}}}{a(t)} \longrightarrow 0(t \rightarrow T)$, then

$$
\liminf _{t \rightarrow T}\|u(t)\|_{L^{2}(|x|<a(t))} \geq\|Q\|_{L^{2}},
$$

where $Q$ is a ground state solution of (1.3). 
(ii) For any $\varepsilon>0$, there exists a $K>0$ such that

$$
\liminf _{t \rightarrow T}\|u(t)\|_{L^{2}\left(|x|<K\left(\frac{T-t}{\ln \mid \ln (T-t)}\right)^{\frac{1}{2}}\right)}>(1-\varepsilon)\|Q\|_{L^{2}} .
$$

Remark 1. From Tsutsumi [19], we can get that

$$
\liminf _{t \rightarrow T}\|u(t)\|_{L^{2}\left(|x|<K(T-t)^{1 / 2}\right)}>(1-\varepsilon)\|Q\|_{L^{2}} .
$$

Obviously the result of this paper is superior to that of [19].

Before the proof of Theorem 3.1 we give several lemmas.

Lemma 3.2 (Strauss [18). Assume that $N \geq 2$. Let $V(x)$ be a radially symmetric function in $H^{1}\left(\mathbb{R}^{N}\right)$. Then for any $R>0$,

$$
\|V\|_{L^{\infty}(|x|>R)}^{2} \leq C_{0} R^{-(N-1)}\|\nabla V\|_{L^{2}(|x|>R)}\|V\|_{L^{2}(|x|>R)},
$$

where $C_{0}$ does not depend on $R$ and $V(x)$.

Lemma 3.3 (Weinstein [20]). For any $f \in H^{1}\left(\mathbb{R}^{N}\right)$,

$$
\|f\|_{2+4 / N}^{2+4 / N} \leq(1+2 / N)\left(\frac{\|f\|_{2}}{\|Q\|_{2}}\right)^{4 / N}\|\nabla f\|_{2}^{2}
$$

where $Q$ is the unique ground state solution of equation (1.3).

In order to state our proof precisely, we need an auxiliary function $\rho(x)$ : let $\rho(x)=\rho(|x|)$ be a radially symmetric nonnegative function in $C_{0}^{1}\left(\mathbb{R}^{N}\right)$ such that

$$
\rho(r)= \begin{cases}1, & r=|x|<1 / 2 \\ 0, & r=|x|>1\end{cases}
$$

and $0 \geq \rho_{r}(r) \geq-8$.

Lemma 3.4. Let $N \geq 2$, and let $u(t)$ be a radially symmetric solution of the Cauchy problem (1.1), (1.2) in $C([0, T) ; H)$ such that $u(t)$ blows up at a finite time T. We put $\lambda(t)=\|\nabla u(t)\|_{2}$. Then, there exist two positive constants $M_{1}$ and $M_{2}$ such that

$$
\limsup _{t \rightarrow T} \frac{\|\nabla u(t)\|_{2}}{\|\nabla u(t)\|_{L^{2}\left(|x|<M_{1} / \lambda(t)\right)}} \leq M_{2} .
$$

Proof. Let $M_{1}$ be a large positive constant to be determined later. From (2.2), we have

$$
\begin{aligned}
\lambda(t)^{2} & \leq \frac{N}{N+2}\left(\left\|\rho\left(\frac{\lambda(t)}{M_{1}} x\right) u(t)\right\|_{2+4 / N}+\left\|\left\{1-\rho\left(\frac{\lambda(t)}{M_{1}} x\right)\right\} u(t)\right\|_{2+4 / N}\right)^{2+4 / N}+E\left(u_{0}\right) \\
& \leq C\left\|\rho\left(\frac{\lambda(t)}{M_{1}} x\right) u(t)\right\|_{2+4 / N}^{2+4 / N}+C\left\|\left\{1-\rho\left(\frac{\lambda(t)}{M_{1}} x\right)\right\} u(t)\right\|_{2+4 / N}^{2+4 / N}+E\left(u_{0}\right),
\end{aligned}
$$

where $\rho(x)$ is defined as in (3.4). 
The Gagliardo-Nirenberg inequality implies that

$$
\begin{aligned}
& \left\|\rho\left(\frac{\lambda(t)}{M_{1}} x\right) u(t)\right\|_{2+4 / N}^{2+4 / N} \\
\leq & C\left\|\rho\left(\frac{\lambda(t)}{M_{1}} x\right) u(t)\right\|_{2}^{4 / N}\left\|\nabla\left\{\rho\left(\frac{\lambda(t)}{M_{1}} x\right) u(t)\right\}\right\|_{2}^{2} \\
\leq & C\|u(t)\|_{2}^{4 / N}\left\|\nabla \rho\left(\frac{\lambda(t)}{M_{1}} x\right) \cdot u(t)+\rho\left(\frac{\lambda(t)}{M_{1}} x\right) \cdot \nabla u(t)\right\|_{2}^{2} \\
\leq & C\|u(t)\|_{2}^{4 / N}\left\|\nabla \rho\left(\frac{\lambda(t)}{M_{1}} x\right) \cdot u(t)\right\|_{2}^{2}+C\|u(t)\|_{2}^{4 / N}\left\|\rho\left(\frac{\lambda(t)}{M_{1}} x\right) \cdot \nabla u(t)\right\|_{2}^{2} \\
\leq & C\|u(t)\|_{2}^{4 / N}\left\|\frac{\lambda(t)}{M_{1}} \cdot \nabla \rho(x) \cdot u(t)\right\|_{2}^{2}+C\|u(t)\|_{2}^{4 / N}\|\nabla u(t)\|_{L^{2}\left(|x|<\frac{M_{1}}{\lambda(t)}\right)}^{2} \\
\leq & C\|u(t)\|_{2}^{4 / N} \frac{\lambda(t)^{2}}{M_{1}^{2}}\|u(t)\|_{2}^{2}+C\|u(t)\|_{2}^{4 / N}\|\nabla u(t)\|_{L^{2}\left(|x|<\frac{M_{1}}{\lambda(t)}\right)}^{2} \\
\leq & \frac{C\|u(t)\|_{2}^{2+4 / N}}{M_{1}^{2}} \lambda(t)^{2}+C\|u(t)\|_{2}^{4 / N}\|\nabla u(t)\|_{L^{2}\left(|x|<\frac{M_{1}}{\lambda(t)}\right)}^{2} .
\end{aligned}
$$

By Lemma 3.2, we have

$$
\begin{aligned}
& C\left\|\left\{1-\rho\left(\frac{\lambda(t)}{M_{1}} x\right)\right\} u(t)\right\|_{2+4 / N}^{2+4 / N} \\
\leq & \|u(t)\|_{L^{2+4 / N}\left(\left|\frac{\lambda(t)}{M_{1}} x\right|>\frac{1}{2}\right)}^{2+4 / N}\|u(t)\|_{L^{2}\left(|x|>\frac{M_{1}}{2 \lambda(t)}\right)}^{2} \\
\leq & C\|u(t)\|_{L^{\infty}\left(|x|>\frac{M_{1}}{2 \lambda(t)}\right)}^{4 / N}\left\|^{2 / N}\right\|^{2+2 / N} \\
\leq & C\left(\frac{\lambda(t)}{M_{1}}\right)^{2(N-1) / N}\|\nabla u(t)\|_{L^{2}\left(|x|>\frac{M_{1}}{2 \lambda(t)}\right)}^{2+u(t) L_{L^{2}\left(|x|>\frac{M_{1}}{2 \lambda(t)}\right)}} \\
\leq & C\left(\frac{\lambda(t)}{M_{1}}\right)^{2(N-1) / N} \lambda(t)^{2 / N}\|u(t)\|_{L^{2}\left(|x|>\frac{M_{1}}{2 \lambda(t)}\right)}^{2(2)} \\
\leq & \frac{C\|u(t)\|_{2}^{2 / 2 / N}}{M_{1}^{2(N-1) / N}} \lambda(t)^{2} .
\end{aligned}
$$

From (3.6)-(3.8), we have

$$
\begin{aligned}
\lambda(t)^{2} \leq & C_{1}\|u(t)\|_{2}^{4 / N}\|\nabla u(t)\|_{L^{2}\left(|x|<\frac{M_{1}}{\lambda(t)}\right)}^{2} \\
& +\left\{\frac{C_{2}\|u(t)\|_{2}^{2+4 / N}}{M_{1}^{2}}+\frac{C_{3}\|u(t)\|_{2}^{2+2 / N}}{M_{1}^{2(N-1) / N}}\right\} \lambda(t)^{2}+E\left(u_{0}\right) .
\end{aligned}
$$

If we choose $M_{1}$ so large that

$$
\frac{C_{2}\|u(t)\|_{2}^{2+4 / N}}{M_{1}^{2}}+\frac{C_{3}\|u(t)\|_{2}^{2+2 / N}}{M_{1}^{2(N-1) / N}} \leq \frac{1}{2},
$$

then we have by (3.10),

$$
\lambda(t)^{2} \leq 2 C_{1}\|u(t)\|_{2}^{4 / N}\|\nabla u(t)\|_{L^{2}\left(|x|<\frac{M_{1}}{\lambda(t)}\right)}^{2}+2 E\left(u_{0}\right) .
$$

Since $\lambda(t) \rightarrow \infty(t \rightarrow \infty)$, (3.11) and (2.1) imply that

$$
\|\nabla u(t)\|_{L^{2}\left(|x|<M_{1} / \lambda(t)\right)} \longrightarrow \infty(t \rightarrow T) .
$$

This fact and (3.11) show (3.5).

Now, we are in the position to prove Theorem 3.1 .

Proof of Theorem 3.1. Let $\rho(x)$ be defined as in (3.4). We put $\rho_{a}(x)=\rho\left(\frac{x}{a(t)}\right)$ and $\lambda_{a}(t)=\left\|\nabla\left(\rho_{a} u_{b}(t)\right)\right\|_{2}$. 
(i) By (2.2) and Lemma 3.2, we have

$$
\begin{aligned}
\lambda(t)^{2} & -\frac{N}{N+2}\|u(t)\|_{L^{2+4 / N}\left(|x|<\frac{a(t)}{2}\right)}^{2+4 / N} \\
& =\frac{N}{N+2}\|u(t)\|_{L^{2+4 / N}\left(|x|>\frac{a(t)}{2}\right)}^{2+4 / N}+E\left(u_{0}\right) \\
& \leq \frac{N}{N+2}\|u(t)\|_{L^{\infty}\left(|x|>\frac{a(t)}{2}\right)}^{4 / N}\|u(t)\|_{L^{2}\left(|x|>\frac{a(t)}{2}\right)}^{2}+E\left(u_{0}\right) \\
& \leq C a(t)^{-2(N-1) / N}\|u(t)\|_{L^{2}\left(|x|>\frac{a(t)}{2}\right)}^{2+2 / N} \lambda(t)^{2 / N}+E\left(u_{0}\right) .
\end{aligned}
$$

A simple calculation gives us

$$
\begin{aligned}
-\frac{N}{N+2}\left\|\rho_{a} u(t)\right\|_{2+4 / N}^{2+4 / N} \leq-\frac{N}{N+2}\|u(t)\|_{L^{2+4 / N}\left(|x|<\frac{a(t)}{2}\right)}^{2+4 / N} \\
\lambda_{a}(t)^{2} \leq\left(\left\|\rho_{a} \nabla u_{0}\right\|_{2}+\left\|\nabla \rho_{a} \cdot u_{0}\right\|_{2}\right)^{2} \\
\leq\left(\lambda(t)+\frac{C\|u(t)\|_{2}}{a(t)}\right)^{2} \\
\leq \lambda(t)^{2}+\frac{C\|u(t)\|_{2} \lambda(t)}{a(t)}+\frac{C\|u(t)\|_{2}^{2}}{a(t)^{2}}
\end{aligned}
$$

On the other hand, from Lemma 3.3. we have

$$
\frac{N}{N+2}\left\|\rho_{a} u_{b}(t)\right\|_{2+4 / N}^{2+4 / N} \leq\left(\frac{\left\|\rho_{a} u_{b}(t)\right\|_{2}}{\|Q\|_{2}}\right)^{4 / N} \lambda_{a}(t)^{2} .
$$

By (3.12)-(3.15), we obtain

$$
\begin{aligned}
1-\left(\frac{\rho_{a} u_{b}(t)}{\|Q\|_{2}}\right)^{4 / N} \leq & C\|u(t)\|_{2}^{2+2 / N} \frac{\lambda(t)^{2 / N}}{\lambda_{a}(t)^{2 / N}\left(a(t) \lambda_{a}(t)\right)^{2(N-1) / N}} \\
& +\frac{E\left(u_{0}\right)}{\lambda_{a}(t)^{2}}+\frac{C\|u(t)\|_{2}^{2} \lambda(t)}{a(t) \lambda_{a}(t)^{2}}+\frac{C\|u(t)\|_{2}^{2}}{\left(a(t) \lambda_{a}(t)\right)^{2}} .
\end{aligned}
$$

Since $\frac{\left(\frac{\ln |\ln (T-t)|}{T-t}\right)^{\frac{1}{2}}}{a(t)} \longrightarrow 0(t \rightarrow T)$, by Proposition 2.3, we know that

$$
\frac{1}{\lambda(t) a(t)} \rightarrow 0 \quad \text { as } \quad t \rightarrow T
$$

Letting $t \rightarrow T$ in (3.16), we obtained by (2.1), Lemma 3.4 and (3.17) that

$$
\limsup _{t \rightarrow T}\left\{1-\left(\frac{\left\|\rho_{a} u_{b}(t)\right\|_{2}}{\|Q\|_{2}}\right)^{4 / N}\right\} \leq 0,
$$

which proves Theorem 3.1(i).

(ii) We use the same argument as in the proof of Theorem 3.1(i) to prove Theorem 3.1(ii). 
Let $K$ be a sufficiently large constant to be determined later. If we insert $a(t)=$ $\frac{K}{\lambda(t)}$ into (3.16), we have by Lemma 3.4,

$$
\begin{aligned}
& 1-\left(\left\|\rho\left(\frac{\lambda(t)}{K} x\right) u(t)\right\|_{2} /\|Q\|_{2}\right)^{4 / N} \\
& \quad \leq C\|u(t)\|_{2}^{2+2 / N} K^{-2(N-1) / N}+E\left(u_{0}\right) \lambda(t)^{-2}+C\|u(t)\|_{2} K^{-1}+C\|u(t)\|_{2}^{2} K^{-2} .
\end{aligned}
$$

Letting $t \rightarrow T$ in (3.19), we obtain

$$
\begin{aligned}
& \limsup _{t \rightarrow T}\left\{1-\left(\left\|\rho\left(\frac{\lambda(t)}{K} x\right) u(t)\right\|_{2} /\|Q\|_{2}\right)^{4 / N}\right\} \\
& \quad \leq C\|u(t)\|_{2}^{2+2 / N} K^{-2(N-1) / N}+C\|u(t)\|_{2} K^{-1}+C\|u(t)\|_{2}^{2} K^{-2} .
\end{aligned}
$$

(3.20) implies that if we choose $K$ sufficiently large, then (3.2) holds.

\section{REFERENCES}

1. J. Bourgain and W. Wang, Construction of blowup solutions for the nonlinear Schrödinger equation with critical nonlinearity, Ann. Sc. Norm. Super. Pisa Cl. Sci. 25(1997), 197-215. MR:1655515 (99m:35219)

2. T. Cazenave, An introduction to nonlinear Schrödinger equations, Textos de metodos mathmatics, 22 Rio de Janeiro, 1989.

3. J. Ginibre and G. Velo, On a class of nonlinear Schrödinger equations, J. Funct. Anal. 32(1979), 1-71. MR.533219 (82c:35058)

4. R. T. Glassy, On the blowing up of solutions to the Cauchy problem for nonlinear Schrödinger equations, J. Math. Phys. 18 (1977), 1794-1797. MR0460850(57:842)

5. M. K. Kwong, Uniqueness of positive solutions of $\triangle u-u+u^{p}=0$ in $\mathbb{R}^{N}$, Arch. Rational Mech. Anal. 105(1989), 243-266. MR969899 (90d:35015)

6. M. J. Landman, G. C. Papanicolaou, C. Sulem, and P. L. Sulem, Rate of blowup for solutions of the nonlinear Schrödinger equation at critical dimension, Phys. Rev. A 38(1988), 38373843. MR 966356 (89k:35218)

7. F. Merle and P. Raphaël, Sharp upper bound on the blow-up rate for the critical nonlinear Schrödinger equation, Geom. Funct. Anal. 13(2003), 591-642. MR1995801 (2005j:35207)

8. F. Merle and P. Raphaël, On universality of blow-up profile for $L^{2}$ critical nonlinear Schrödinger equation, Invent. Math. 156(2004), 565-672. MR2061329(2006a:35283)

9. F. Merle and P. Raphaël, The blow-up dynamic and upper bound on the blow-up rate for critical nonlinear Schrödinger equation, Annals of Math. (2)161 (2005), 157-222. MR 2150386 (2006k:35277)

10. F. Merle and P. Raphaël, Profiles and quantization of the blow up mass for critical nonlinear Schrödinger equation, Comm. Math. Phys. 253(2005), 675-704. MR2116733(2006m:35346)

11. F. Merle and P. Raphaël, On a sharp lower bound on the blow-up rate for the $L^{2}$ critical nonlinear Schrödinger equation, J. Amer. Soc. 19(2005), 37-90. MR2169042 (2006j:35223)

12. F. Merle and Y. Tsutsumi, $L^{2}$-concentration of blow-up solutions for the nonlinear Schrödinger equation with critical power nonlinearity. J. Diff. Eqs. 84(1990), 205-214. MR:1047566 (91e:35194)

13. H. Nawa, Asymptotic and limiting profiles of blowup solutions of the nonlinear Schrödinger equation with critical power, Comm. Pure Appl. Math. 52(1999), 193-270. MR1653454 (99m:35235)

14. G. C. Perelman, On the blow-up phenomenon for the critical nonlinear Schrödinger equation in 1D, Ann. Henri Poincaré 2(2001), 605-673.

15. P. Raphaël, Stability of log-log bound for blow up solutions to the critical nonlinear Schrödinger equation, Math. Ann. 331(2005), 577-609. MR.2122541 (2006b:35303)

16. T. Ogawa and Y. Tsutsumi, Blow-up for $\mathrm{H}^{1}$ solution for the nonlinear Schrödinger equation, J. Diff. Eq. 92(1991), 317-330. MR1120908 (92k:35262) 
17. T. Ogawa and Y. Tsutsumi, Blow-up for $H^{1}$ solution for the nonlinear Schrödinger equation with critical power nonlinearity, Proc. Amer. Math. Soc. 111(1991), 487-496 MR.1045145 (91f:35026)

18. W. A. Strauss, Existence of solitary waves in higher dimensions, Comm. Math. Phys. 55(1977), 149-162. MR0454365 (56:12616)

19. Y. Tsutsumi, Rate of concentration of blow-up solutions for the nonlinear Schrödinger equation with critical power, Nonlinear Anal. T. M. A. 15 (1990), 719-724. MR.1074950 (91j:35043)

20. M. I. Weinstein, Nonlinear Schrödinger equations and sharp interpolation estimates, Comm. Math. Phys. 87(1983), 567-576. MR691044 (84d:35140)

21. J. Zhang, Sharp conditions of global existence for nonlinear Schrödinger and Klein-Gordon equations, Nonlinear Anal. T. M. A. 48(2002), 191-207. MR.1870752 (2002h:35303)

Software Laboratory, Sichuan Normal University, Chengdu 610066, People's RepubLIC OF CHINA

Current address: College of Mathematics and Software Science, Sichuan Normal University, Chengdu, 610066, People's Republic of China

E-mail address: lixiaoguang1235@msn.com

College of Mathematics and Software Science, Sichuan Normal University, Chengdu, 610066, People's Republic of China 